\title{
TRECE MUJERES BACHILLERES EN HUMANIDADES EN EL SIGLO XIX: PIONERAS O ENTROMETIDAS EN LA UNIVERSIDAD DE CHILE ${ }^{1}$
}

\author{
Ariadna Biotti Silva
}

1. Los documentos reproducidos en estas páginas son custodiados en el Fondo Histórico de la Universidad de Chile del Archivo Central Andrés Bello de la Universidad de Chile. Las versiones completas se encuentran disponibles en la biblioteca digital y el sitio web del Archivo Central Andrés Bello. En el caso de Manuela Cormatches y Eloísa Cuadra, los documentos corresponden a Actas de Sesión del Consejo Universitario, custodiadas también por el Archivo Central Andrés Bello. 


\section{ARIADNA BIOT'TI SILVA}

Licenciada en Historia de la Universidad de Chile (2005). Máster en Histoire, Ecole des Hautes Etudes en Sciences Sociales (2009). Doctora en Historia y Civilizaciones, Ecole des Hautes Etudes en Sciences Sociales (2014). Doctora en Historia mención Historia de Chile, Universidad de Chile (2014). Sus áreas de interés dicen relación con la historia del libro y la lectura, cultura escrita, impresa en Chile y el mundo occidental. Se ha desempeñado como profesora en la Universidad Metropolitana de Ciencias de la Educación. Actualmente es coordinadora del Área de Investigación Patrimonial del Archivo Central Andrés Bello de la Universidad de Chile. 


\section{TRECE MUJERES BACHILLERES EN HUMANIDADES EN EL SIGLO XIX: PIONERAS O ENTROMETIDAS EN LA UNIVERSIDAD DE CHILE}

Las universidades se fundaron como instituciones masculinas y así permanecieron hasta la segunda mitad del siglo XIX. Deriva de ello el hecho de que estos espacios no sean ambientes donde se puedan relacionar fácilmente hombres y mujeres, cuestión que aumenta su complejidad si consideramos la aceptación del feminismo y la diversidad sexual en sus aulas. Tal es así, que en las construcciones no se consideraban baños para mujeres, siendo el nuevo edificio de la Facultad de Derecho de nuestra Universidad (inaugurado en 1938) el primero en incluirlos en su diseño original, aunque sólo para las estudiantes pues las académicas aparecerían después. La primera titulada en Ingeniería en 1919, Justicia Espada Acuña, no iba al baño durante toda su jornada de estudio.

La historia de la exclusión de las mujeres de los espacios intelectuales en la cultura occidental es bastante larga, pues el miedo a la diferencia respecto del modelo hegemónico de la masculinidad en el poder y de la heteronormatividad de la sexualidad signó en el cuerpo de las mujeres temores que estructuran un imaginario vigente: deficiente, incompleto, carnal, impuro, sensual y atractivo hasta la lascivia. El cristianismo demonizó a la mujer atribuyéndole pasiones bestiales condenables. Se explicaba entonces que las mujeres fueran subordinadas al hombre desde el principio de la creación pues todas compartían el infortunio del pecado de Eva.

"De esta manera, en Occidente se excluye a las mujeres del mundo del intelecto y se las destina a las tareas inferiores e inferiorizantes del cuidado y la domesticidad; de esta forma, se crea un espacio de exclusividad masculina en la dedicación a la vida eclesiástica que, en ese momento, es el espacio por excelencia para el desarrollo de las aptitudes intelectuales"

2. Buquet Corleto, Ana; Moreno, Hortensia; Cooper Jenifer; Mingo, Araceli. Intrusas en la universidad. Universidad Nacional Autónoma de México, Programa Universitario de Estudios de Género, Instituto de Investigaciones sobre la Universidad y la Educación, Torre del IISUE, Centro Cultural Universitario. México. 2013. P. 27 
Esa tradición cultural europea fue heredada a las universidades latinoamericanas y no resulta ajena al desarrollo histórico que ha tenido la Universidad de Chile, la institución de educación superior republicana más antigua del país, que a lo largo de sus 176 años ha desarrollado todo un campo de conocimiento para legitimar en la dialéctica del mérito académico personal a una serie de sujetos varones posicionados como los más inteligentes del país, los que cumplen su función y misión social, otorgándole a la educación una apariencia progresista comprometida con la serie de ideales abstractos que pregona la modernidad. Estos ideales se manifiestan en la trama de un discurso políticamente correcto que va avanzando acorde a los contextos y las políticas estatales.

En el campo cristiano laico y conservador que era la universidad no fue sino hasta el último tercio del siglo XIX cuando una cantidad crítica de mujeres exigió su ingreso a la universidad. Para cumplir su objetivo, ellas debieron derribar una serie de muros y barreras, afrontando dificultades rutinarias y cotidianas pues se trataba de un espacio de lo más incierto para las mujeres. No era fácil la educación para las mujeres de ese tiempo pues todo indicaba que su lugar natural era el hogar y el "ser para" la familia, ya sea en condición de hija, madre o esposa. La mujer representaba el honor del núcleo o linaje doméstico. De ahí que estar en lo público sin la representación de un varón podía ser visto como un descaro, un viso de inmoralidad interpretable como sinónimo de conducta libertina, inmoral, una osadía o una clara insinuación sexual latente. Un ejemplo de ello es que hasta el día de hoy en el imaginario común las palabras mujer pública o profesional son sinónimos de prostituta.

No existían colegios mixtos y eran muy pocas las profesoras mujeres educadas para afrontar la vida universitaria. Existían contados colegios femeninos, los cuales no necesariamente impartían la formación necesaria para obtener el grado de bachillerato que se exigía para entrar como estudiante superior. Tampoco los colegios masculinos eran lugares idóneos para la presencia femenina. En consecuencia, las mujeres estudiantes eran fundamentalmente autodidactas, autoinstruidas, aprendían de sí mismas, de su propia experiencia siendo maestras y estudiantes al mismo tiempo. Buscaban el conocimiento interactuando directamente con el saber, lo cual debió significar una experiencia de aprendizaje distinta, una relación con el conocimiento menos frontal, más libre, personal, independiente. En el autoaprendizaje, los roles del maestro con el educando se invierten constantemente. La mujer autodidacta pudo enseñar mientras aprendía.

Por tales motivos es tan importante la labor que hicieran dos maestras: Antonia Ignacia Tarragó González, directora del Colegio de Santa Teresa, quien en 1872 había planteado la importancia social de la instrucción de la mujer, y la profesora Isabel Le Brun de Pinochet, directora del Colegio de la Recoleta, quien tres años después planteó el tema al Consejo Universitario. Cabe recordar que desde 1850 
hasta 1967 para entrar a la Universidad de Chile se exigía haber obtenido el grado de bachiller, es decir, un diploma o distinción académica de inspiración francesa creado por Napoleón en 1808, hasta el día de hoy vigente y conocido como el Baccalaureate, cuyo objetivo era la selección de postulantes para la continuación de estudios universitarios.

A juicio de José Vera, la prueba era inicialmente en humanidades, incorporándose posteriormente la de matemáticas para entrar a la carrera de Ingeniería. La prueba de bachillerato consistía en una parte oral y otra escrita. La escrita era una traducción del latín de autores clásicos grecorromanos y una composición castellana sobre temas correspondientes a materias propias del currículo. Para rendirlo, el estudiante debía tener completa la enseñanza secundaria completa. Según el investigador, se solicitaba también un certificado de buena conducta otorgado por el liceo, institución que también corresponde a los inicios del siglo XIX y sobre la cual la Universidad de Chile ejercía el rol de Superintendencia de Educación ${ }^{3}$.

El examen era rendido exclusivamente por varones. Un cambio importante ocurrió el 6 de febrero 1877, cuando gracias a la gestión de las profesoras Antonia Ignacia Tarragó González e Isabel Le Brun de Pinochet fue aprobado el conocido Decreto Amunátegui, que estipuló:

"Que conviene estimular a las mujeres a que hagan estudios serios y sólidos;

Que ellas pueden ejercer con ventaja algunas de las profesiones denominadas científicas;

Que importa facilitarles los medios de que puedan ganar la subsistencia por sí mismas;

\section{Decreto:}

Se declara que las mujeres deben ser admitidas a rendir exámenes válidos para obtener títulos profesionales con tal que ellas se sometan para ello a las mismas disposiciones a que están sujetos los hombres.

Comuníquese y publíquese"”.

3. El Sistema de Admisión a la Universidad: Permanencia y Cambio. Entrevista al autor del libro El Sistema de Admisión a la Universidad: Permanencia y Cambio, del historiador José Vera Rodríguez. Disponible en: http://www.psu.demre.cl/videos/16-libro-bachillerato-jose-vera. Consultado por última vez el 26 de agosto de 2018.

4. Archivo Nacional de Chile. Decreto Amunátegui - Mujeres a la Universidad. Disponible en: http:// www.archivonacional.cl/sitio/Contenido/Institucional/8046:DECRETO-AMUNATEGUIMUJERES-A-LA-UNIVERSIDAD. Consultado por última vez el 26 de agosto de 2018. 
De esta forma se explica cómo el 11 de abril de 1881 el rector de la universidad le confiriera el grado de bachiller a Eloísa Rita Díaz Insunza, egresada del colegio de Isabel Le Brun 5 . En 1886 pasó a ser la primera mujer titulada de Chile y Sudamérica, "quien tuvo que combatir con energías las preocupaciones sociales que desde el primer día de sus estudios dificultaron la realización de su ideal. En el curso de los seis años de sus estudios, fue acompañada diariamente por su señora madre a la Universidad, a los hospitales y a la Escuela de Medicina".

Según los estudios de José Vera, paralelamente habría ingresado a la universidad Juana Sáez Pineda a estudiar Artes, carrera que dependía de la Facultad de Filosofía y Humanidades, la cual no requería rendir examen de bachillerato?

El 16 de mayo aprueba el examen Celinda Carvajal, de quien no hemos encontrado más datos ${ }^{8}$. En 1882, Pilar Pérez Molina ${ }^{9}$, quien se titula como médico en $1894^{10}$.

En 1883, Ernestina Pérez y Barahona obtiene el grado ${ }^{11}$. De acuerdo con sus biógrafos, Ernestina hizo, al igual que Eloísa, sus primeros estudios en el liceo de la Señora Isabel Le Brun Pinochet. Fue una aventajada alumna, al extremo de ser adelantada en varias materias en la Escuela de Medicina, lugar donde se tituló como médico cirujano dos días después de Eloísa Díaz. Obtuvo numerosos premios, siendo enviada a París por el Estado de Chile para proseguir sus estudios de ginecología y medicina infantil y doméstica. Fue una luchadora infatigable contra

5. Bachillerato en Humanidades de Eloísa Díaz. Santiago: 11 de abril de 1881. Fondo Histórico de la Universidad de Chile. Tomo A-J, sin foliar. Archivo Central Andrés Bello. Universidad de Chile.

6. Figueroa, Pedro Pablo. Diccionario biográfico general de Chile, Imprenta Victoria, Santiago, 1888, p. 164.

7. Vera Rodríguez, José. (2015). El sistema de admisión a la universidad: Permanencia y cambio, 1842-1973 (1a. ed.). Santiago de Chile: Universitaria, p.108.

8. Bachillerato en Humanidades de Celinda Carvajal. Santiago: 13 de mayo de 1881. Fondo Histórico de la Universidad de Chile. Tomo A-J, sin foliar. Archivo Central Andrés Bello. Universidad de Chile.

9. Bachillerato en Humanidades de Pilar Pérez Molina Santiago: 20 de abril de 1882. Fondo Histórico de la Universidad de Chile. Tomo L-Z, sin foliar. Archivo Central Andrés Bello. Universidad de Chile.

10. Anales de la Universidad de Chile. Tomo LXXXIX. Boletín de Instrucción Pública. Imprenta Cervantes. Sesión del Consejo Universitario. 21 de diciembre de 1894.

11. Bachillerato en Humanidades de Ernestina Pérez y Barahona. Santiago: 5 de enero de 1883. Fondo Histórico de la Universidad de Chile. Tomo L-Z, sin foliar. Archivo Central Andrés Bello. Universidad de Chile. 
la tuberculosis y el alcoholismo, comprometiéndose con la educación sanitaria popular, obrera y sindical ${ }^{12}$.

También en 1883 se gradúo con el mismo grado de bachiller a Euridice Pinochet Le Brun, hija de la educadora Isabel Le Brun y Marcos Fidel Pinochet ${ }^{13}$. Se desempeñó como profesora de castellano en el colegio dirigido por su madre. Su hermana Noemi Pinochet Le Brun, profesora de música de la misma institución, obtuvo el grado el 5 de abril de $1887^{14}$.

No encontramos más mujeres bachilleras hasta el 21 de marzo de 1887, cuando alcanzó la distinción Matilde Throup Sepúlveda ${ }^{15}$, primera abogada de Chile y pionera del Derecho, quien obtuvo el grado de Licenciada en Leyes en 1892.

Más tarde, en noviembre de 1887 obtuvo el grado de bachiller Zoila Rosa Sotomayor Reveco, de quien no tenemos más datos académicos ${ }^{16}$. Dos años más tarde se les concede el grado habilitante a cinco mujeres:

- Eva Quezada Acharán: obtuvo el grado de bachiller en $1890^{17}$. Licenciado en Medicina y Farmacia, titulada posteriormente como médico cirujano en $1894^{18}$. Fue funcionaria pública y cumplió diversas misiones de gobierno en Europa ${ }^{19}$.

12. Empresa Periodística Chile. Diccionario biográfico de Chile. Cuarta edición. Talleres gráficos La Nación, Santiago, 1942, p.488- 489.

13. Bachillerato en Humanidades de Euridice Pinochet Le Brun. Santiago: 3 de enero de 1883. Fondo Histórico de la Universidad de Chile. Tomo L-Z, sin foliar. Archivo Central Andrés Bello. Universidad de Chile.

14. Figueroa, Pedro Pablo, Diccionario biográfico general de Chile, Imprenta Victoria, Santiago, 1888, p.428. Bachillerato en Humanidades de Noemi Pinochet Le Brun. Santiago: 5 de abril de 1887. Fondo Histórico de la Universidad de Chile. Tomo J-Z, sin foliar. Archivo Central Andrés Bello. Universidad de Chile.

15. Bachillerato en Humanidades de Matilde Throup Sepúlveda. Santiago: 24 de marzo de 1887. Fondo Histórico de la Universidad de Chile. Tomo J-Z; sin foliar. Archivo Central Andrés Bello. Universidad de Chile.

16. Bachillerato en Humanidades de Zoila Sotomayor Reveco. Santiago: 14 de noviembre de 1887. Fondo Histórico de la Universidad de Chile. Tomo J-Z, sin foliar. Archivo Central Andrés Bello. Universidad de Chile.

17. Bachillerato en Humanidades de Eva Quezada Acharán. Santiago: 25 de abril de 1889. Fondo Histórico de la Universidad de Chile. Tomo M-Z, sin foliar. Archivo Central Andrés Bello. Universidad de Chile.

18. Anales de la Universidad de Chile. Tomo LXXXIX. Boletín de Instrucción Pública. Imprenta Cervantes. Sesión del Consejo Universitario. P.177.

19. Virgilio Figueroa. Diccionario histórico biográfico y bibliográfico de Chile. Establecimientos gráficos Balcells \& Co. Santiago, Tomo IV y V. 1931. P.581. 
- Amantina Quezada Acharán ${ }^{20}$.

- Glafiria Vargas ${ }^{21}$.

- Manuela Cormatches Roca ${ }^{22}$.

- Eloísa Cuadra Iglesias ${ }^{23}$.

Salvo en el caso de Eva Quezada, desconocemos las historias académicas de las egresadas como bachilleres. Es probable que esto se deba a que su desarrollo haya derivado a campos no calificados dentro de la institucionalidad universitaria oficial. Nos referimos a artistas, escritoras y profesoras que realizando actividades intelectuales, no fueron ni han sido consideradas como parte reconocida o habilitada por la institución para cumplir el ejercicio de una profesión que certificaba la capacidad o rendimiento profesional en el campo de su ejercicio social y público.

Las primeras mujeres egresadas fueron Licenciados en Medicina y Farmacia, tituladas como médico cirujano tal como ocurría en Europa. Las mujeres se integraron a la vida universitaria en un proceso lento, complejo y violento en lo cotidiano. Nos referimos a problemas quizás para muchos triviales como el señalado al inicio, ir al baño, entrar a una sala de clases repleta de hombres, comer, vestirse para ir, conversar, etc.

A 137 años del primer bachillerato sabemos que los números de escolaridad universitaria femenina han ido aumentando, pero el sueño de la equidad de género está lejos de ser alcanzado pues prevalece un continuum que apela consciente o inconscientemente a la naturaleza biológica especista y a la superioridad constitutiva del hombre por sobre la mujer.

La tortuosa historia de la exclusión de las mujeres de los ámbitos de la educación formal tiene que ver con la violencia de género incrustada en las universidades y especialmente en el aula como espacio donde se pueden manifestar o representar sesgos y prejuicios. Las mujeres siguen siendo tratadas como "minorías", tienen menos posibilidades de contratación, se les pagan salarios más bajos y tienen poca participación en cargos directivos, puestos de prestigio y de poder. Pioneras o

20. Bachillerato en Humanidades de Amantina Quezada Acharán. Santiago: 25 de abril de 1889. Fondo Histórico de la Universidad de Chile. Tomo M-Z, sin foliar. Archivo Central Andrés Bello. Universidad de Chile.

21. Bachillerato en Humanidades de Glafiria Vargas. Santiago: 29 de abril de 1889. Fondo Histórico de la Universidad de Chile. Tomo M-Z, sin foliar. Archivo Central Andrés Bello. Universidad de Chile.

22. Acta de sesión del Consejo de la Universidad de Chile. 6 de mayo de 1889, p.117. Archivo Central Andrés Bello.

23. Acta de sesión del Consejo de la Universidad de Chile. 4 de noviembre de 1889, p.323. Archivo Central Andrés Bello. Universidad de Chile. 
entrometidas, las mujeres que exigen sus derechos son vistas hoy como "pintiparadas" o inadecuadas, parecidas o semejantes a otro, dependientes, endemoniadas o monstruosas, el desarrollo de la costilla de Adán, igualadas sin mérito con una cosa parecida que no es sí misma sino una intromisión o un descaro de ser, estar y exigir respeto ${ }^{24}$.

24. "Hay profesores que buscan más que una relación de amistad con las alumnas, pero a ellas (las denunciantes) yo las veo muy pintiparadas. Dando declaraciones de acá para allá. Yo no las vi muy destruidas psicológicamente". Declaración del profesor Premio Nacional de Historia Gabriel Salazar. "El día que Gabriel Salazar apoyó a profesores sancionados de acoso sexual y abuso de poder en la U. de Chile”. El Desconcierto. 27.12.2016. Consultado el 22 de julio de 2018: http://www.eldesconcierto.cl/2016/12/27/el-dia-que-gabriel-salazar-apoyo-aprofesores-acusados-de-acoso-sexual-y-abuso-de-poder-en-la-u-de-chile/ 


\section{REFERENCIAS}

Archivo Nacional de Chile. Decreto Amunátegui - Mujeres a la Universidad. Disponible en: http://www.archivonacional.cl/sitio/Contenido/ Institucional/8046:DECRETO-AMUNATEGUI-MUJERES-A-LAUNIVERSIDAD. Consultado por última vez el 26 de agosto de 2018.

Buquet Corleto, Ana; Moreno, Hortensia; Cooper, Jenifer; Mingo, Araceli. Intrusas en la universidad. Universidad Nacional Autónoma de México, Programa Universitario de Estudios de Género, Instituto de Investigaciones sobre la Universidad y la Educación, Torre del IISUE, Centro Cultural Universitario. México. 2013. P.27.

"El día que Gabriel Salazar apoyó a profesores sancionados de acoso sexual y abuso de poder en la U. de Chile”. El Desconcierto. 27.12.2016. Consultado el 22 de julio de 2018: http://www.eldesconcierto.cl/2016/12/27/el-dia-que-gabrielsalazar-apoyo-a-profesores-acusados-de-acoso-sexual-y-abuso-de-poder-enla-u-de-chile/

El Sistema de Admisión a la Universidad: Permanencia y Cambio. Entrevista al autor del libro El Sistema de Admisión a la Universidad: Permanencia y Cambio, del historiador José Vera Rodríguez: Disponible en: http://www.psu.demre.cl/videos/16-librobachillerato-jose-vera. Consultado por última vez el 26 de agosto de 2018.

Empresa Periodística Chile. Diccionario biográfico de Chile. Cuarta edición. Talleres gráficos La Nación, Santiago, 1942.

Figueroa, Pedro Pablo. Diccionario biográfico general de Chile, Imprenta Victoria, Santiago, 1888.

Vera Rodríguez, José (2015). El sistema de admisión a la universidad: Permanencia y cambio, 1842-1973 (1a. ed.). Santiago de Chile: Universitaria.

Virgilio Figueroa. Diccionario histórico biográfico y bibliográfico de Chile. Establecimientos gráficos Balcells \& Co. Santiago, Tomo IV y V. 1931. 
TRECE MUJERES BACHILLERES EN HUMANIDADES EN EL SIGLO XIX: PIONERAS O ENTROMETIDAS EN LA UNIVERSIDAD DE CHILE 
TRECE MUJERES BACHILLERES EN HUMANIDADES EN EL SIGLO XIX: PIONERAS O ENTROMETIDAS EN LA UNIVERSIDAD DE CHILE
Ariadna Biotti Silva

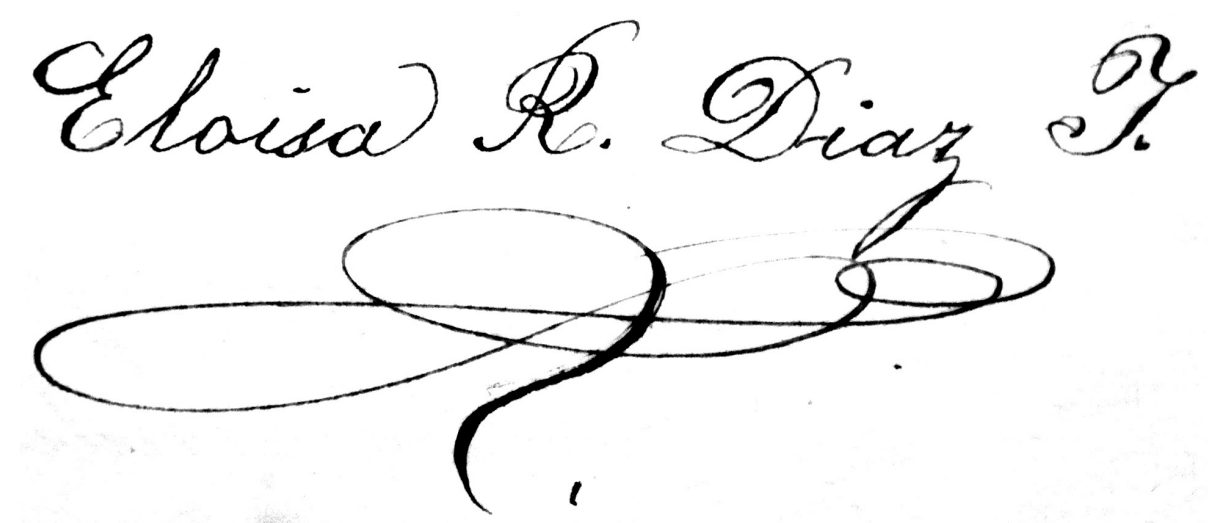


Heicar fector de la Anivenisas

eftoabiencto zencict las x ancenel queve exifin hasa oftar el quado de bachiltes en to facilleted de filosofiar hu? mandadef.

Inia pechtico se visualhedis informe a las rectares delf Inptituto Vacio

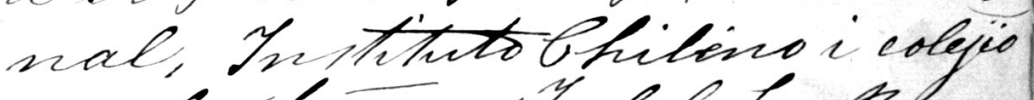
de la tevisa Irabel be Bsun de timerhet.
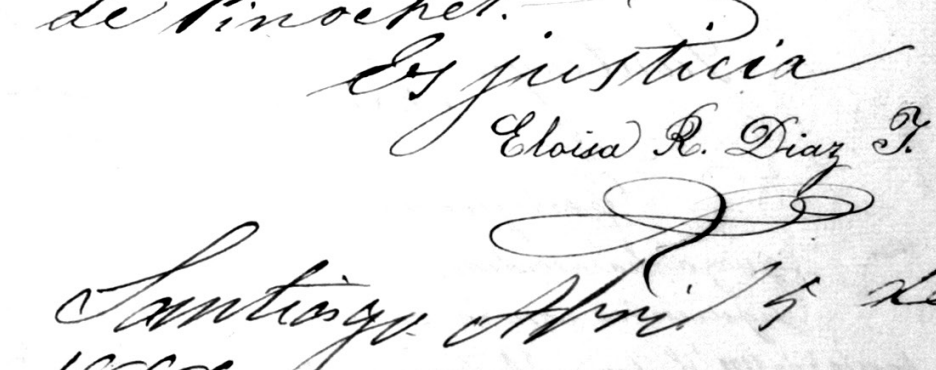
1887

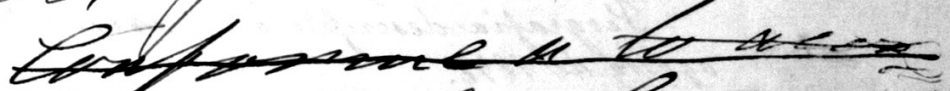

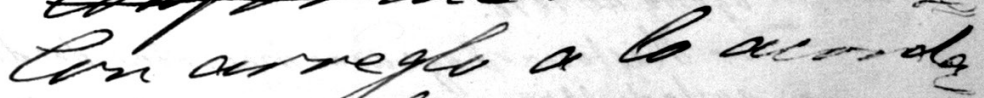

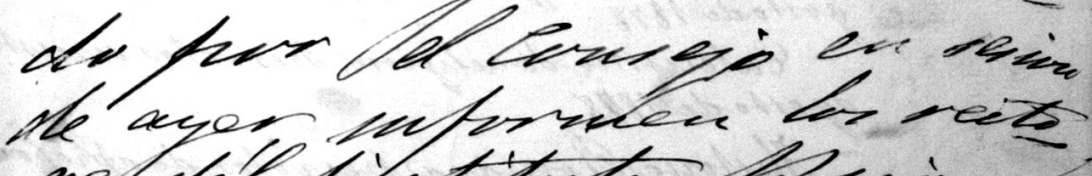

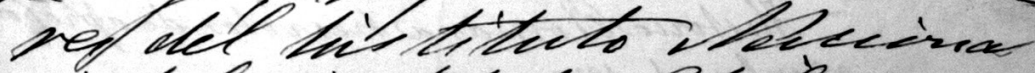

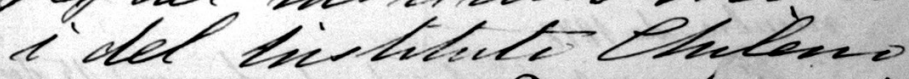

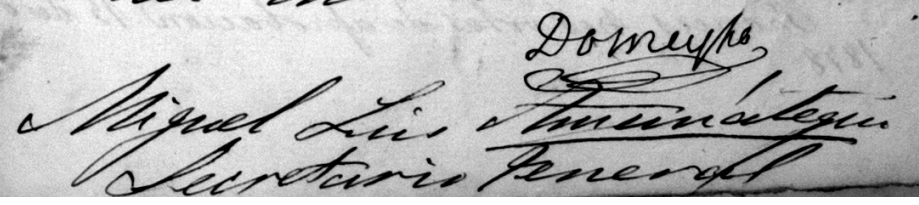


TRECE MUJERES BACHILLERES EN HUMANIDADES EN EL SIGLO XIX: PIONERAS O ENTROMETIDAS EN LA UNIVERSIDAD DE CHILE Ariadna Biotti Silva

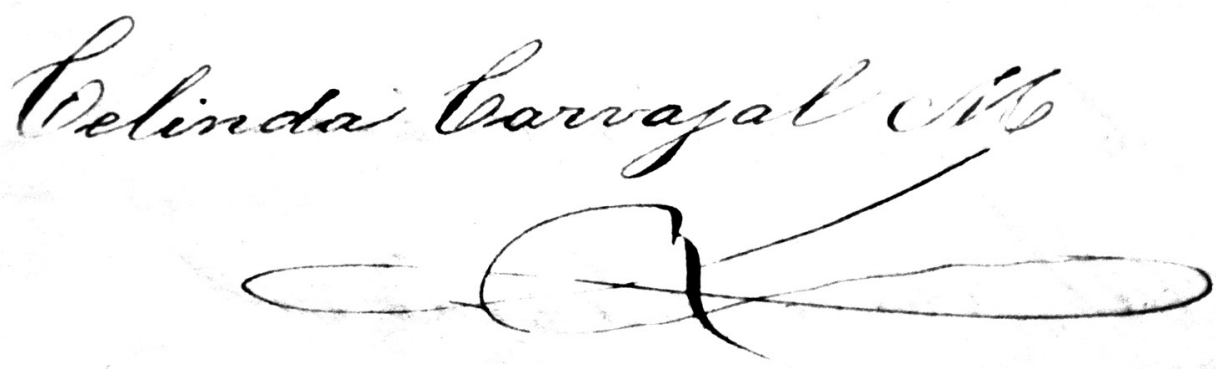

164 
REVISTA ANALES

Séptima serie. No 14/2018

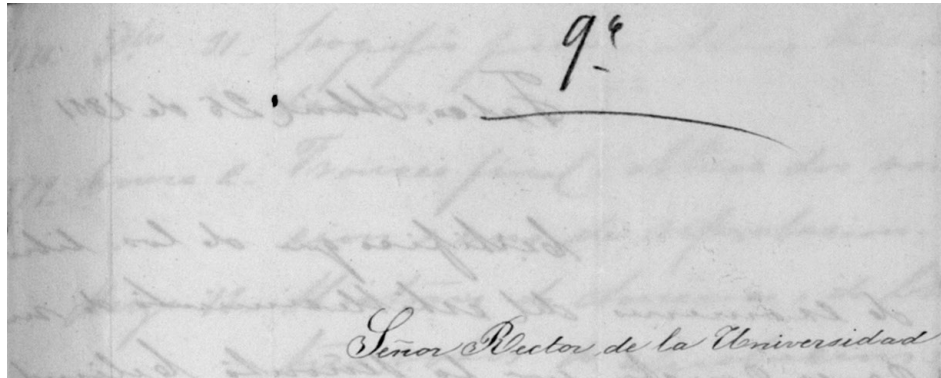

belinda bavragel a Xes resfuluesamente digo que deseanda buser ot grado de bacticler en la faculaded de filos ofia i humarridades i habienda cumplido con los nequisitos que se exijen fara ello.

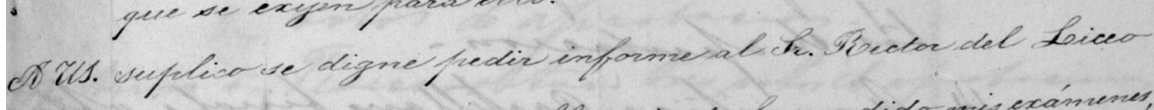
de Falea en uryo estatbinuiento he rendido mis exámenes, i evacuado i frevios los trapinites de extelo, concederme el - Wituto qie solicits:

Celinda barragal cit

ita

cice ole pouba.

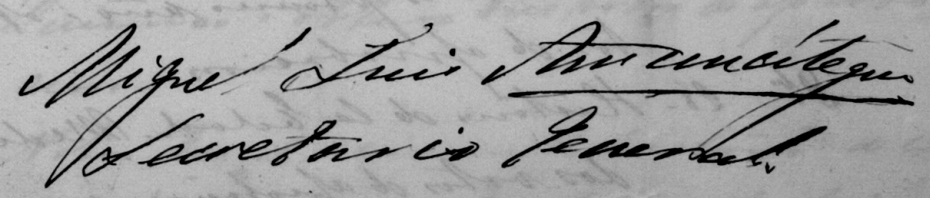

165 
Pilou Permolle 
REVISTA ANALES

Séptima serie. $\mathrm{N}^{\circ} 14 / 2018$

0,0 do abir cert82

Penor Rectos.

Hilar Perez Molina, aspirante al grads de bachiller en humanida des, habiendoren dido todos los exámenes que se exijen fearads tar dicho graido

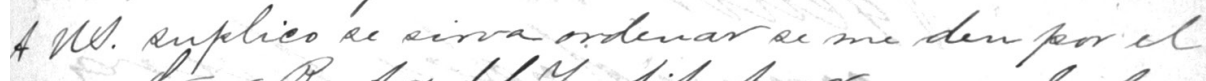
Invor Pector del Mnstitust. sacional las certifieads correapendinites

Dilos Perente

(1) setsof

finfurme of pectis

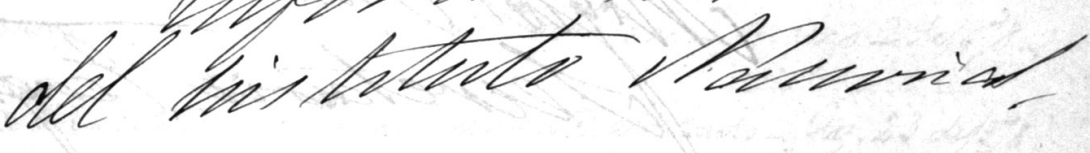

bomeung

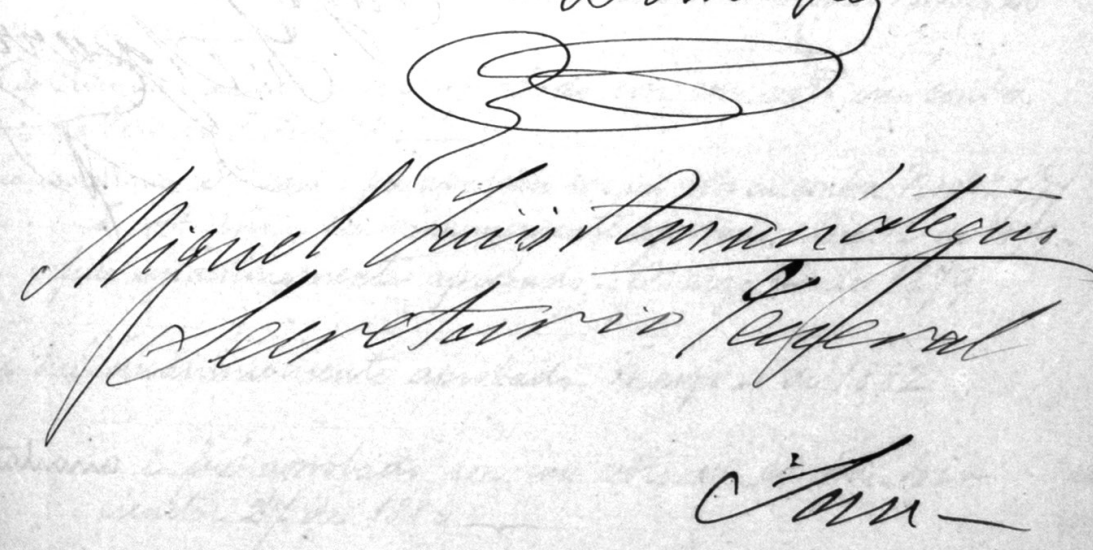

167 


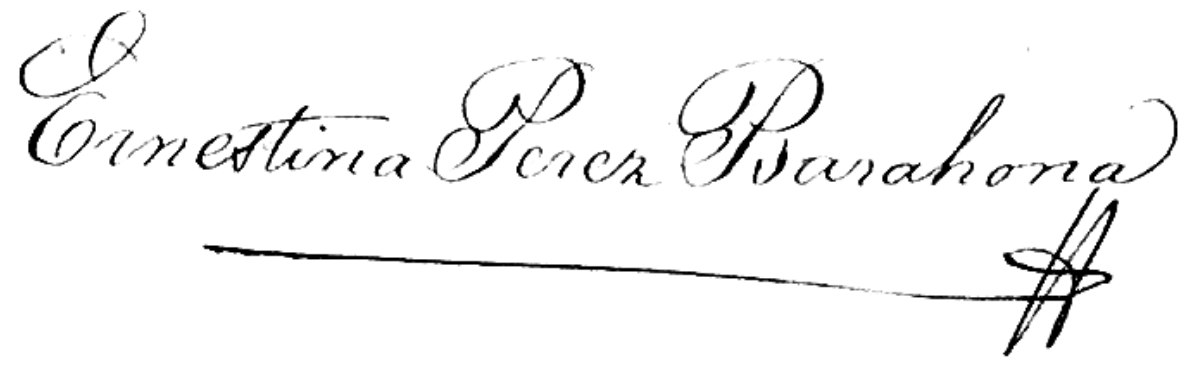


REVISTA ANALES

Séptima serie. No 14/2018

$$
3^{-4 a}
$$

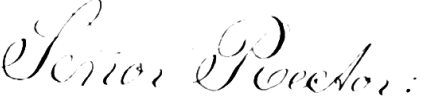

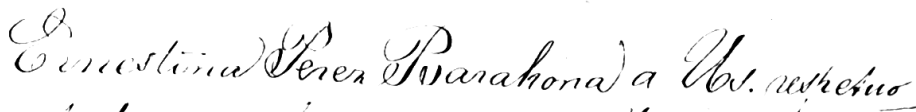

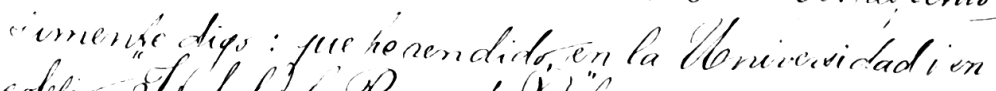

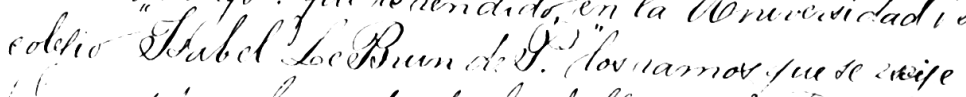

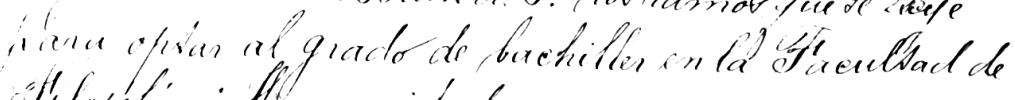

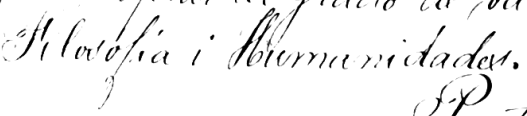

(S) tanto.

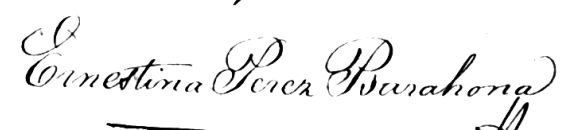

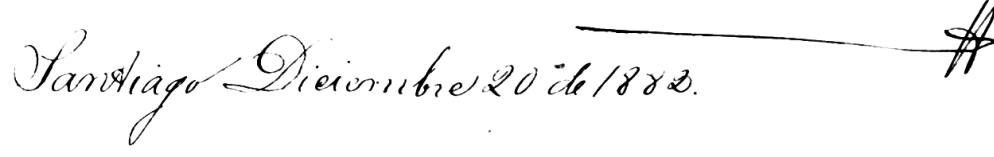

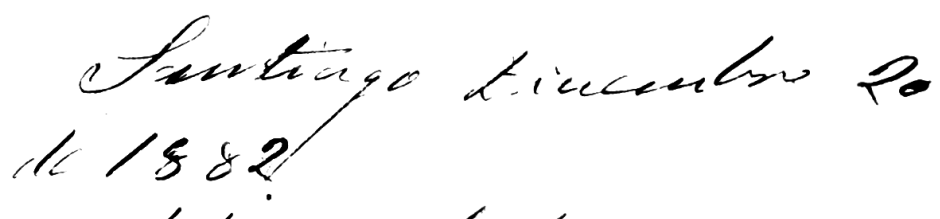

lufpre al feromater de la lluezensidal. Damus

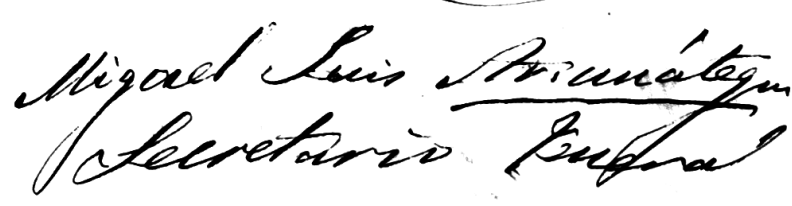

169 
TRECE MUJERES BACHILLERES EN HUMANIDADES EN EL SIGLO XIX: PIONERAS O ENTROMETIDAS EN LA UNIVERSIDAD DE CHILE

Ina Biotti Sili

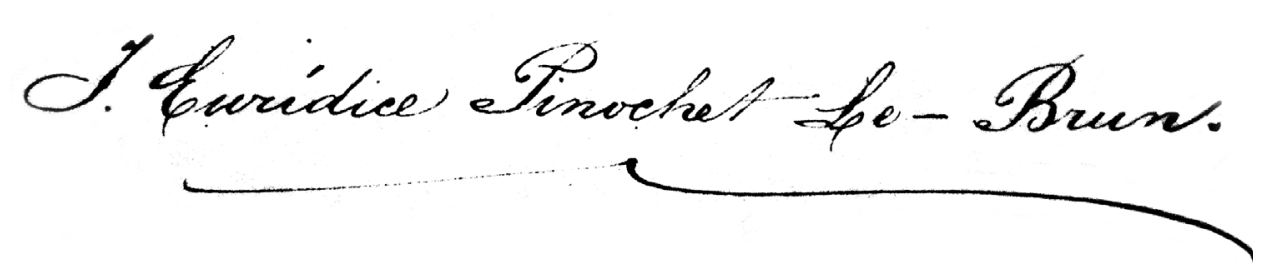

170 
REVISTA ANALES

Séptima serie. $\mathrm{N}^{\circ} 14 / 2018$

Steñor Ructor.

Deseando obterex el titulo de Buchiller en to racultad de Bilosofia i Tha manidades, i hatiendo rendido todos los exámences que

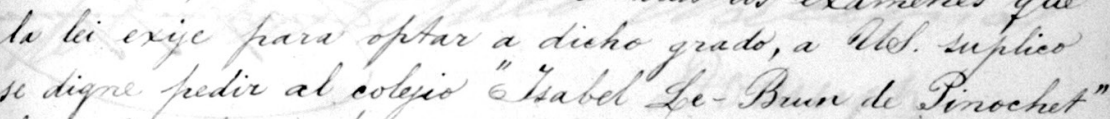
los cerrificados de todos mis cxámenes.

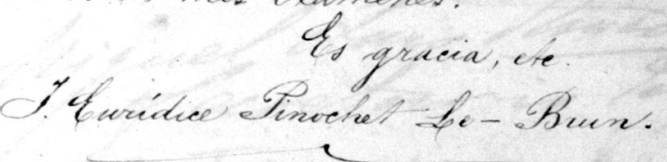

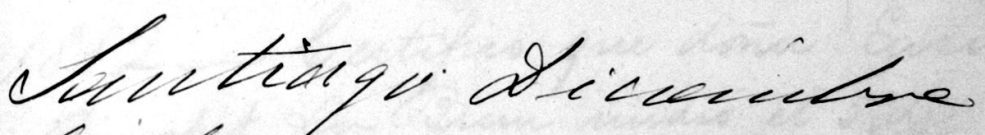
6 de 18is?

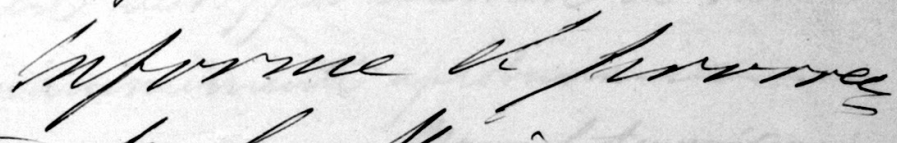

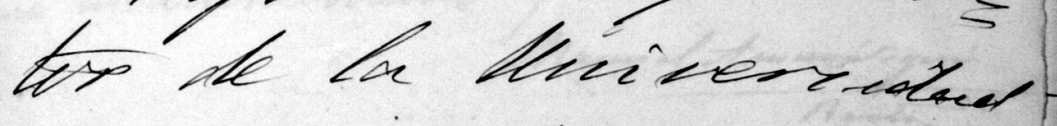

Domeyas

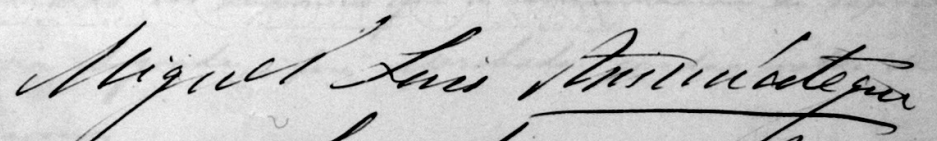

Ale elv. Ruevor de la

Universidad de qhile.

171 


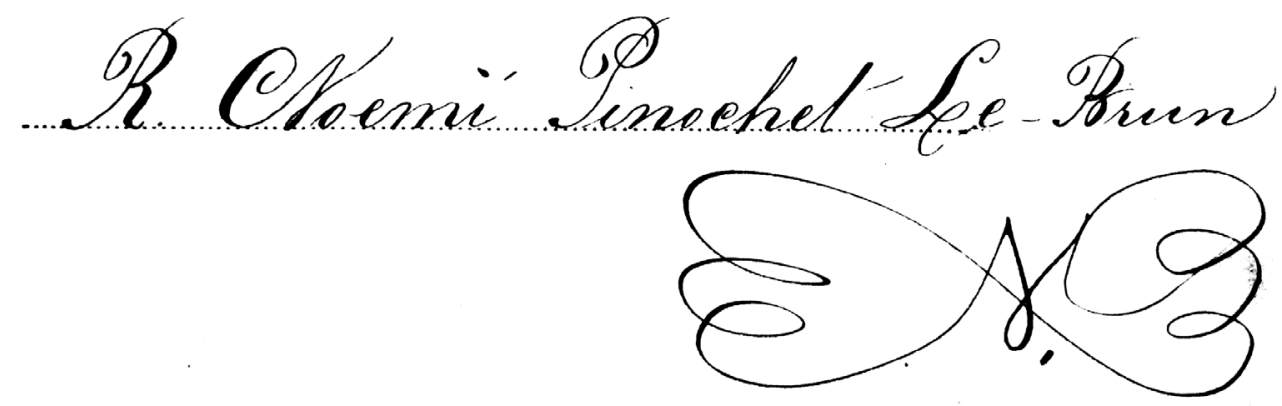




\section{UNIVERSIDAD DE CHILE}

Weñor Prector:

Qeoneagra el 26 de mayo

mis estudios de humanidades en flices

Pinveluet fe ofumen

nacido departamento de

la provincia de "थ...de 18.6\% espongo que, habiendo hecho Sincehe/"..... y rendido todos los examenes exigidos por los reglamentos universitarios en dieno fices en la Otnirersidad

me hallo en el caso de ser admitida á rendir la prueba final para obtener el grado de bachiller en la facultad de filosofia y humanidades.

En esta virtud, pido que en vista de los antecedentes que comprueban mis estudios, se me permita rendir la prueba referida.

Es justicia.
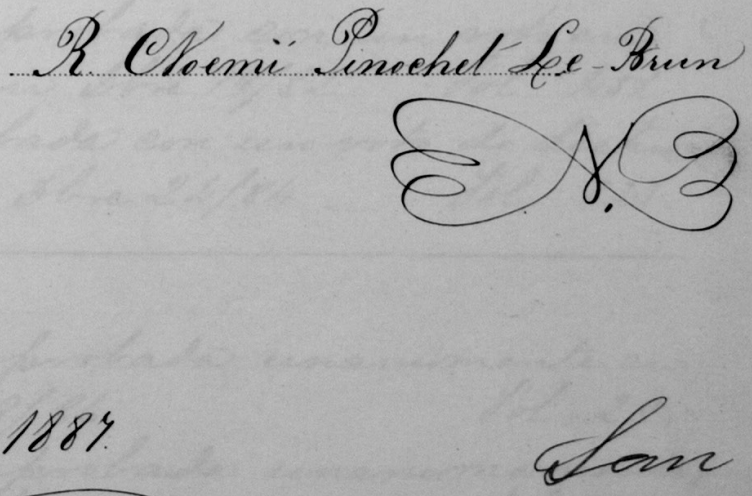


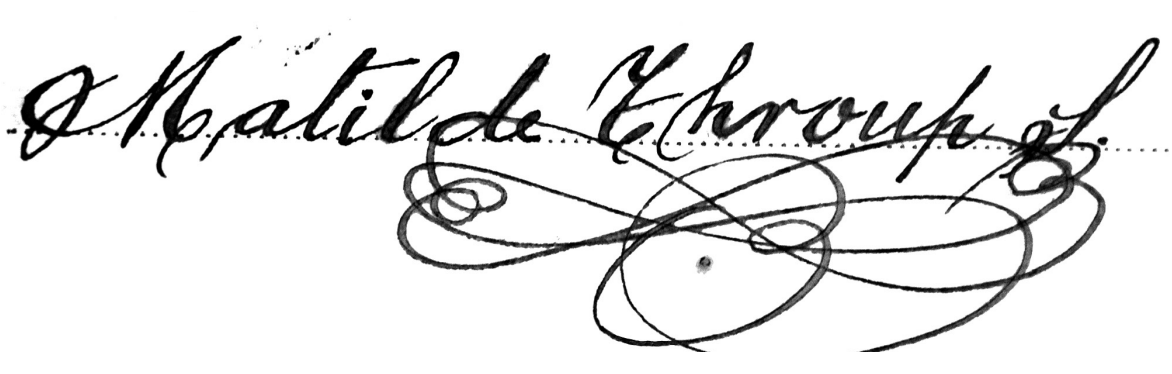


UNIVERSIDAD DE CHILE

Wenor Zुbector:

QHatide Ehroufu glepintueda

en, Ohangal

$\rightarrow 0 r a m$

mis estudios de humanidades en El Colyio "Label Lee- Orumide Tinochet $y$ rendido todos los examenes exigidos por los reglamentos unirersi-

tarios en el miems eatablicimientusi en fa dle nivensidad

me hallo en el caso de ser admitido á rendir la prueba final para obtener el grado de bachiller en la facultad de filosofia y humanidades.

En esta virtud, pido que en vista de los antecedentes que comprueban mis estudios, se me permita rendir la prueba referida.

$$
\text { Es justicia. }
$$

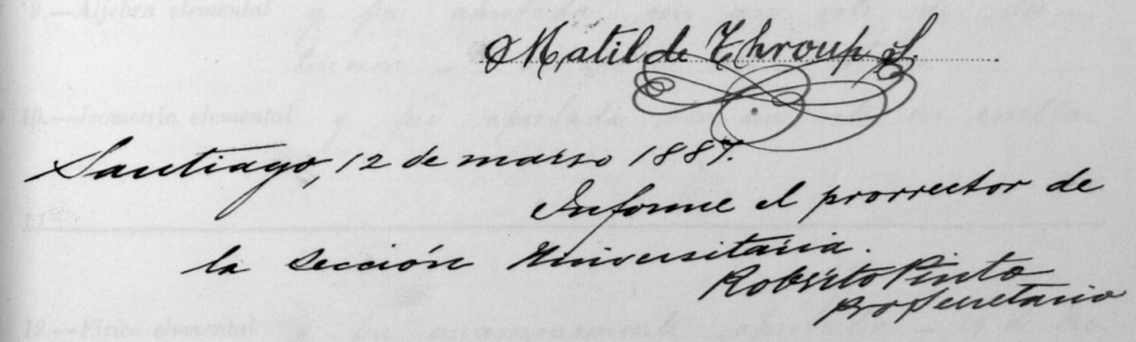




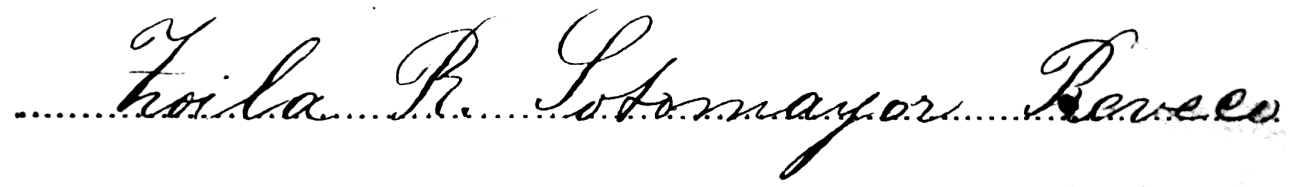


Revista ANALES

Séptima serie. No 14/2018

UNIVERSIDAD DE CHILE

Trever

Geñor Zrector.

Triba Th Sotrmayos Reveco. nacido

en,.... Sam Bernan di el It de ellayo departamento de la Chetosia de 1864 espongo que, habiendo hecho mis estudios de humanidades en en enlyio Isabel Lebresn de Irvachet y rendido todos los examenes exigidos por los reglamentos universitarios en el suissa estableciniento i en la Uoniversidad me hallo en el caso de ser admitido á rendir la prueba final para obtener el grado de bachiller en la facultad de filosofia y humanidades.

En esta virtud, pido que en vista de los antecedentes que comprueban mis estudios, se me permita rendir la prueba referida.

Es justicia.

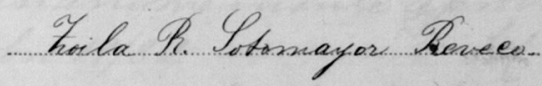

vantiago, 29 de mazo de $188 \%$

ciór Univeritania.

177 
TRECE MUJERES BACHILLERES EN HUMANIDADES EN EL SIGLO XIX: PIONERAS O ENTROMETIDAS EN LA UNIVERSIDAD DE CHILE Ariadna Biotti Silva

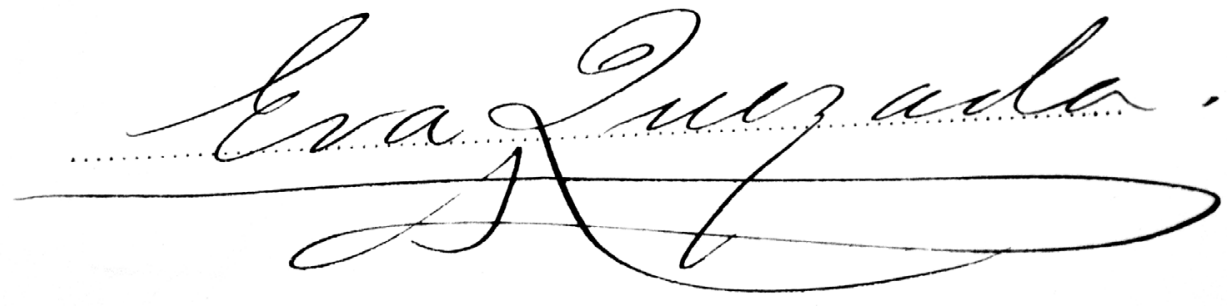

178 
CNILE

\%

SENOR RECTOR:

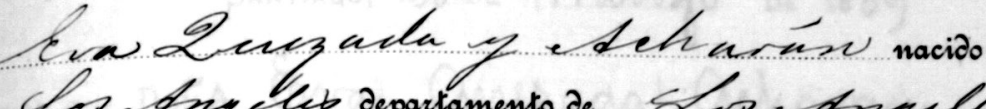

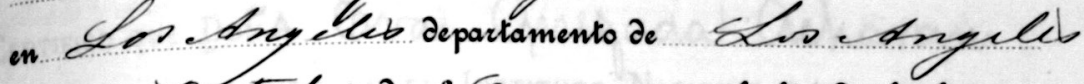
d 2,1 de tetrubo ae 18 Gexpongo: que, habienao hecho mis estrioios secunaarios on e a liclael ole alesses pur forciata

y rendido todos los exámenes exigias por los reglamentos universitarios

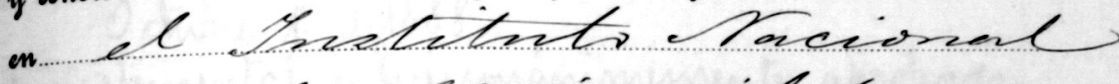

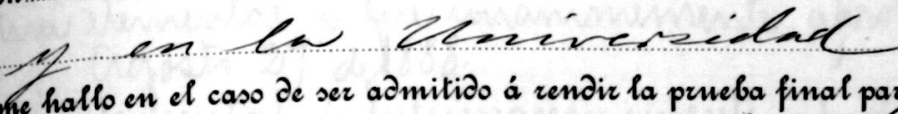
el grado de Bachiller en la Facultad de

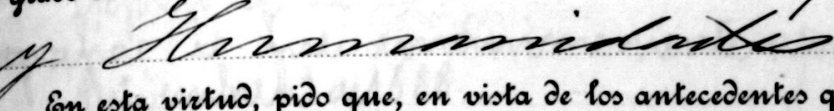

En esta virtua, piao que, en vista de los antecedentes que comprueban mis estudios, se me permita rendir la puneba referida.

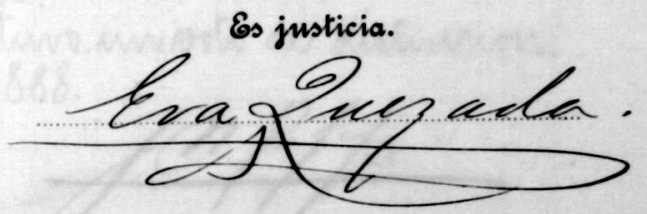

Santiago, If de Allaryo de 1889

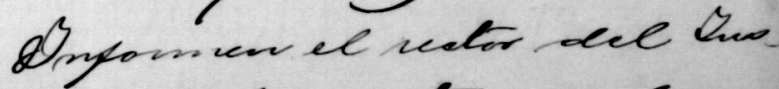

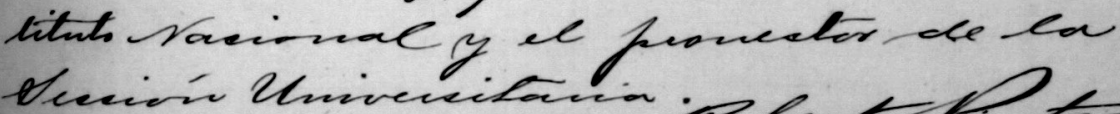


TRECE MUJERES BACHILLERES EN HUMANIDADES EN EL SIGLO XIX: PIONERAS O ENTROMETIDAS EN LA UNIVERSIDAD DE CHILE Ariadna Biotti Silva

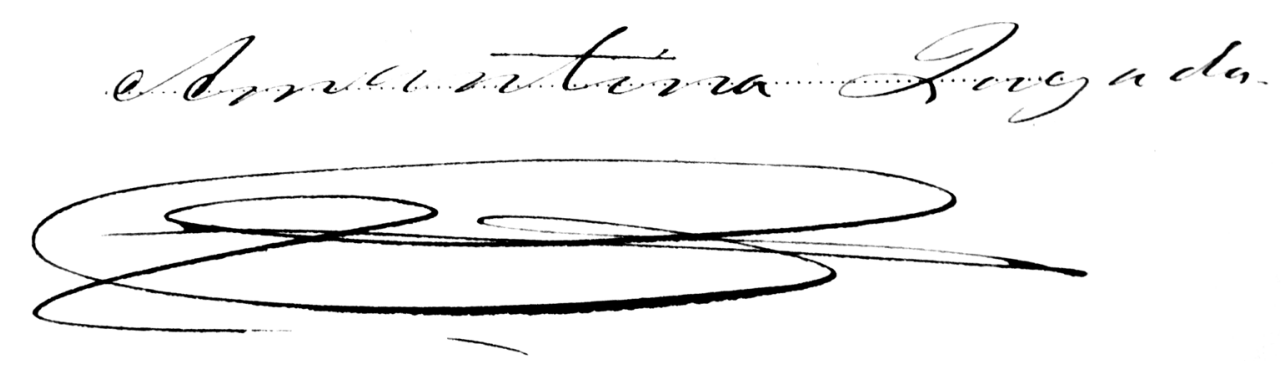

180 
i

SENOR RECTOR:

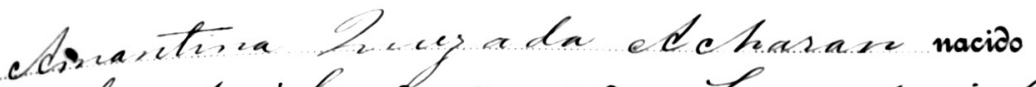

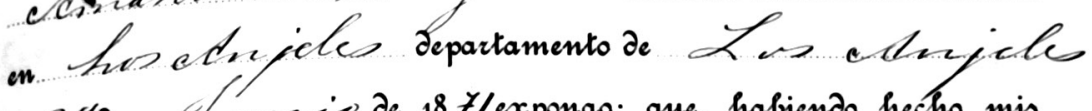

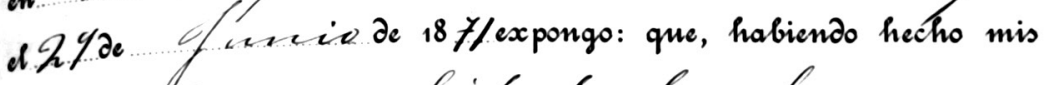

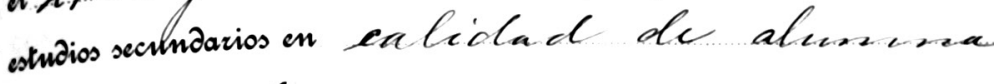
priarada

y rendido todos los exámenes exigiasos por los reglamentos miversitarios

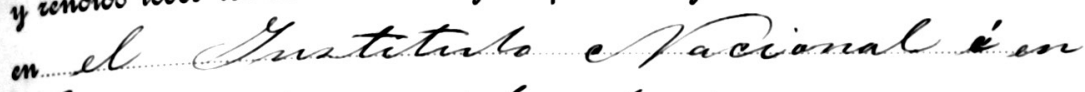
Pa Lcovivespidacl me hallo en el caso de ser admitia á rendir la prueba final para obtener a grado de Bachiller en la facultad de ffila féa... Mhasmaceralaclas

En esta virtud, pido que, en vista de los antecedentes que comprueban mis estudios, se me permita rendir la pueba referiaa.

Es justicia.

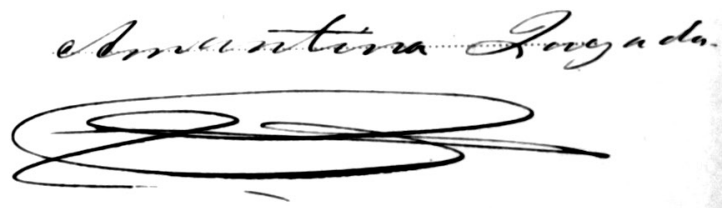

Santiago, 9 de chayso de $18 \times 9$.

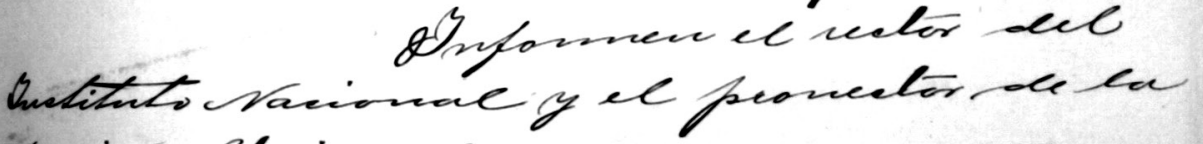
Heción Aluirenitacia.
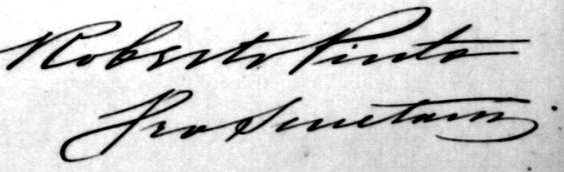
TRECE MUJERES BACHILLERES EN HUMANIDADES EN EL SIGLO XIX: PIONERAS O ENTROMETIDAS EN LA UNIVERSIDAD DE CHILE Ariadna Biotti Silva

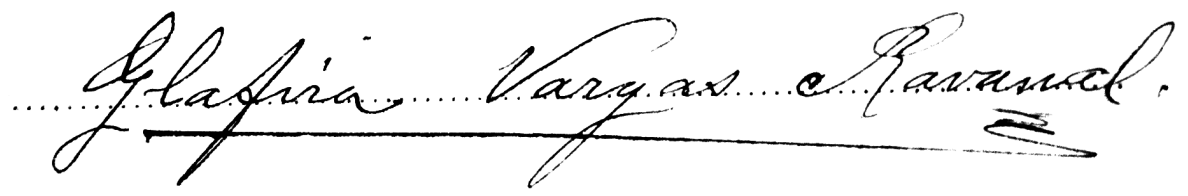

182 
DAD DE CHILE

ir

SENOR Rector:

in.
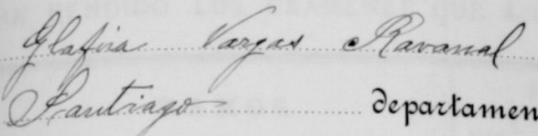

departamento de

nacido

ol 19 de Chariembse de 1870 expongo: que, habiendo hecho mis

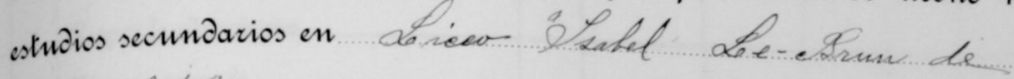
Cineretel.

y rendido todos los exámenes exigidos por los reglamentos universitarios

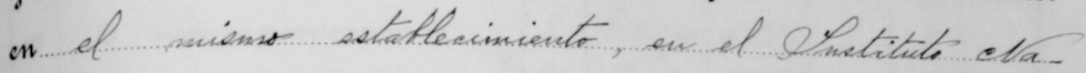
sioud i en ta Rlanivereidat.

me hallo en el caso de ser admitido á rendir la prueba final para obtener

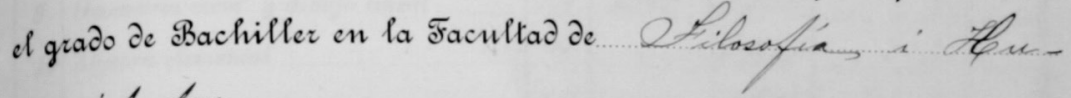
manidadex.

In esta virtud, pido que, en vista de los antecedentes que comprueban mis estudios, se me permita rendir la prueba reperia.

Es justicia.

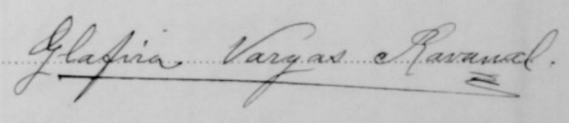

Santiago, 9 de ellarge de $188 \%$

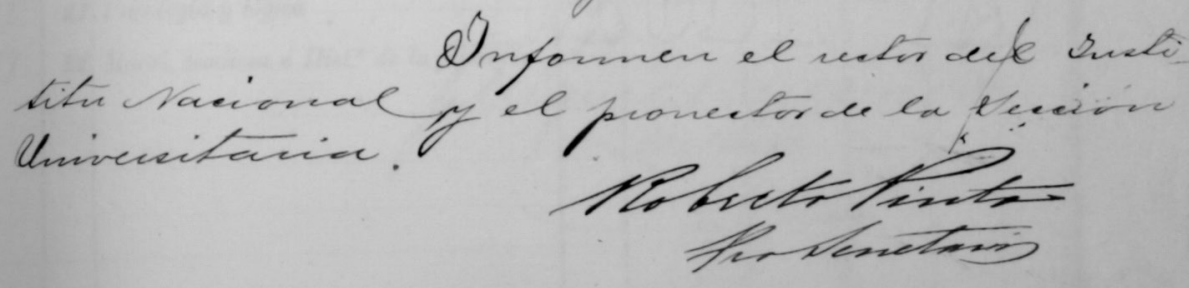


bozenín ge InsTruccíón póblica

CONSEJO DE INSTRUCCIÓN PÚBLICA.-Actas de las sesiones celebradas en los meses de mayo y junio de 1889

\section{Sesión de 6 de mayo de 15s!)}

Fué presidida por el Señor Decano Aguirre y asistieron los Se. ñores Consejeros Asta-Buruaga, Espejo, Letelier, Montt, Prado y el Secretario General que suscribe.

El Señor Decano Barceló envió recado de no poder coneurrir.

Leída y aprobada el acta de la sesión anterior, el Señor Decano Aguirre confirió el grado de Licenciado en Leyes y Ciencias Politicas á Don Nicolás 2. Briceño Campaña, Don Abelardo Contreras Astudillo, Don Eliseo Rojas Polanco y Don Joaquín Gustavo Valledor Sánchez; igual grado en Medicina y Farmacia á Don Benjamín González Lagos, el de Bachiller en Leyes y Ciencias Politicas á Don Luis E. Cifuentes Gromez, Don Rafael Fuenzalida Guzmán, Don Federico Gana Gana, Don Pedro Lamas Benavente y Don Ricardo Sánchez Cruz; igual grado en Medicina y Far. macia á Don Santiago Calderón Azócar, Don José Mauuel Durán Navarro, Don Abraham Rojas Celedón, Don A. Eduardo Sepúlveda Lagos y Don Arturo Toro Osorio; igual grado en Ciencias F'isicas y Matemáticas á Don Carlos García Cross y Don Juan Taulis Muñoz, é igual grado en Filosofia y Humanidades á Doña Manuela Cormatches Roca, Don R. Abraham Campos Car- 


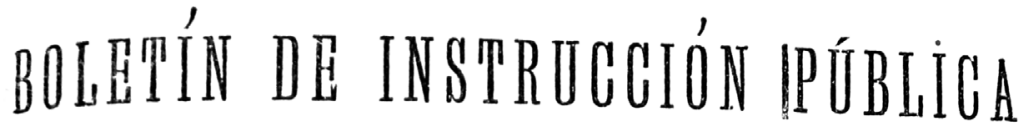

CONSEJO DE INSTRUCCIÓN PÚBLICA.-Actas de las sesiones celebradas en los meses de noviembre y diciembre de 1889

\section{Sesión de 4 de Noviembre de 1899}

Fué presidida por el Señor Don Isidoro Errázuriz, Ministro de Instrucción Pública; y asistieron el Señor Don José Joaquín Aguirre, Rector de la Universidad, los Señores Consejeros AstaBuruaga, Barceló, Barros Brrgoño, Bañados Espinosa, Espejo, Hurtado, Letelier, Montt, Prado y el prosecretario.

El Señor Secretario General envió recado de no poder concurrir, Leída y aprobada el acta de la sesión de 28 de octubre último, el Señor Recior confirió el grado de Bachiller en la Fracultad de Ciencias F'isicas y Matemáticas á don Carlos Cabrera Castillo, Don Francisco Lezaeta Rivas, Don Jorge 2. ${ }^{\circ}$ Pórter Saldivia y Don Arturo Montero Riveros; igual grado en la de Filosofía y Humanidades á Don Alberto Acuña Valdivia, Don Aralio Adriasola Cruz, Don Demetrio Baeza Hidalgo, Doũa Eloisa Cnadra Iglesias, Don Carlos Cossoro Figueroa, Don Arturo Ibáñez Rondizzoni, DonAgustín León Lavín, Don Juan Manuel Ríos Pizarro, Don Brinolfo Sotomayor Gajardo y Don Alberto Zuaznábar Mora. les, a quienes se entregó el correspondiente diploma.

En seguida se dió cuenta.

1. De los siguientes oficios: 